\title{
Asymmetry of training effects in nonspecific transfer of training
}

\author{
JACK E. SHERMAN, LEHA ROBLES, and DAVID R. THOMAS \\ University of Colorado, Boulder, Colorado 80309
}

\begin{abstract}
Three groups of 16 pigeons each were given true discrimination (TD), nondifferential (ND), or single-stimulus (SS) training with line angles, and later were tested for the acquisition of a brightness discrimination. Half of the subjects were tested on the brightness problem immediately after line-angle training; the other half were tested with a wavelength discrimination problem first, then were returned to their original line-angle training regimen, and finally were tested on the brightness problem. Performance on the brightness problem was uninfluenced by experience with a prior wavelength problem. The TD subjects showed both more rapid acquisition and a higher level of asymptotic performance than the SS and ND groups, which did not differ. The TD facilitation effect was due to more rapid extinction of responding to $\mathrm{S}-$. It was argued that failure to obtain an ND retardation effect may be due to the high degree of similarity between training stimuli used in Stage 1 and/or to a test bias against finding ND effects.
\end{abstract}

Thomas and his associates (e.g., Thomas, Freeman, Svinicki, Burr, \& Lyons, 1970) have proposed that, when exposed to true discrimination (TD) or nondifferential (ND) ${ }^{1}$ training involving two stimuli, animals learn about the significance or insignificance of stimuli in general, and not just about the specific training values. This general set, characterized as either an attentiveness or inattentiveness to stimulus differences, may then be reflected in sharper or flatter generalization gradients measured along a dimension which was represented but held constant in original training, or was subsequently experienced. In some generalization studies, a single-stimulus (SS) group provides a theoretically neutral baseline against which the effects of TD and ND training can be assessed. Typically, the SS gradient falls between the sharp TD gradient and the flat ND gradient, although closer to the latter (cf. Bresnahan, 1970; Honig, 1969; Thomas et al., 1970).

The effects of TD, ND, and SS training have also been studied in a transfer of training paradigm. Subjects given TD training acquire a subsequent orthogonal discrimination task more rapidly than SS or ND trained subjects (Eck, Noel, \& Thomas, 1969; Frieman \& Goyette, 1973). This positive transfer has also been attributed to the process of general attention (Eck et al., 1969). Solving a discrimination task may be viewed as, first, attending to the relevant stimuli and, second, attaching the correct responses to these stimuli. An animal which is generally attentive to stimulus differ-

This research was supported by Research Grant HD03486 and Research Service Award MH14617, under the direction of D. R. Thomas. Reprints may be obtained from any of the authors at: Department of Psychology, University of Colorado, Boulder, Colorado 80309. ences should have an advantage over one that is attentively neutral or is inattentive.

The general attention position would predict that, analogous to the relative flattening of slope in generalization following ND training, performance in the acquisition of a new discrimination task should be retarded in ND trained subjects as compared to SS trained subjects. There was only a suggestion in the Eck et al. (1969) study that ND retardation could, in fact, be obtained. Their procedure was divided into four stages: Stages 1 and 3 consisted of TD and ND training with the same stimuli (line angles), whereas Stages 2 and 4 were novel orthogonal transfer tasks (wavelength and brightness dimensions, respectively). An SS group was included in Stages 1 and 2 but was then dropped from the experiment. The authors noted a greater difference in acquisition performance between TD and ND groups on the second transfer task than on the first, which was due to the much poorer performance of the ND group in Stage 4 than in Stage 2. Whether the poorer performance was attributable to the additional training and testing and/or to the nature of the Stage 4 task remains undetermined, since task and amount of training and testing were confounded. Further, the absence of an SS control group in the fourth stage precludes the characterization of the poor performance as an ND retardation effect, since an SS group might have performed equally poorly.

In the present study, we used a brightness discrimination problem like that which produced such poor performance in ND trained subjects in Stage 4 of the Eck et al. (1969) study. However, we eliminated their confound by making the brightness task Stage 2 (i.e., the first transfer problem) for half of our subjects and Stage 4 for the other half. We also included an SS group throughout Stages 2 and 4. 
Several procedural changes from the Eck et al. (1969) study were made in an effort to enhance the likelihood of obtaining an ND retardation effect. In the Eck et al. study, 10-sec blackouts following all stimulus exposures may have served to increase attentiveness in the ND subjects and to attenuate any possible SS-ND differences. Furthermore, the ND trained birds in the Eck et al. study were individually yoked to the TD birds for the number of training sessions they received. This may not have provided enough sessions to produce an optimal ND retardation effect. In the present study, we omitted blackouts and provided all subjects with a substantial number of training sessions in Stage 1 . In the transfer stages of the experiment, we gave 20 sessions of training so that we could examine possible differences in asymptotic performance as well as in acquisition rate.

\section{METHOD}

\section{Subjects}

The subjects were 48 experimentally naive domestic pigeons maintained at $75 \%$ of their free-feeding weights throughout the experiment. Subjects were housed in individual cages where access to grit and water was continuously available.

\section{Apparatus}

Four standard pigeon chambers were used (cf. Hickis, Robles, \& Thomas, in press).

On-key discriminative cues were provided by IEE (Series 0010) in-line display cells mounted $.635 \mathrm{~cm}$ behind the key. Display cells fitted with Kodak Wratten filters No. 74 and No. 99 provided chromatic stimuli with nominal peak wavelength transmission of 538 and $555 \mathrm{~nm}$, respectively. The display cell allowed projection of a white line $.32 \mathrm{~cm}$ wide and $2.5 \mathrm{~cm}$ high on a black surround, in either a vertical (90-deg) position or rotated $30 \mathrm{deg}$ clockwise from vertical, that is, at $60 \mathrm{deg}$. Rear projection of these stimuli on the pecking key was accomplished by the illumination of miniature 14-V lamps (No. 1815).

When discriminative cues were on-key, constant chamber illumination was provided by a $28-\mathrm{V}$ miniature lamp (No. 1829) centered behind a $2.4-\mathrm{cm}$ high strip of white translucent Plexiglas extending the width of the chamber above the intelligence panel. The bright discriminative (off-key) cue consisted of the additional illumination of two $28-V$ ESB bulbs, each $10 \mathrm{~cm}$ to either side of the $28-\mathrm{V}$ lamp. The dim discriminative cue was provided by the illumination of the center lamp in series with an $80-\mathrm{ohm}$ resistor. Even though the Plexiglas strip diffused the light, visual spatial cues were additionally available as a consequence of illuminating the two ESB bulbs (bright stimulus). Measurement of illuminance with a linear photometer (Gamma Scientific, Inc., Model 721) and associated photomultiplier tube (R136) indicated that illuminance in the approximate region of the pigeon's head while pecking was $21.53 \mathrm{~lm} / \mathrm{m}^{2}$ (2 fc) with the bright stimulus, $14.63 \mathrm{~lm} / \mathrm{m}^{2}$ (1.36 fc) with the chamber illumination constant, and $4.95 \mathrm{~lm} / \mathrm{m}^{2}(.46 \mathrm{fc})$ with the dim stimulus.

\section{Procedure}

Preliminary training. On Day 1 , all pigeons were hand shaped to keypeck for $3 \mathrm{sec}$ of access to mixed grain. Following the continuous reinforcement of the first 20 responses, the next 60 reinforcers were earned on a gradually increasing variable interval (VI) schedule of reinforcement until a VI 1-min schedule was in effect. On each of the next two sessions, pigeons earned 30 reinforcers on a constant probability VI 1-min schedule (Catania \& Reynolds, 1968). During this training, the white vertical line was continuously present on the response key.
Subsequent assignment of pigeons to TD, ND, and SS training regimens $(n=16)$ was accomplished by matching their response rates.

Stage 1. During Stage 1 and subsequent stages, subjects received daily training sessions of 30 -min duration. The TD group was given discrimination training with nonsystematically ordered presentations of the vertical white line $(\mathrm{S}+)$ and the 60-deg line (S-). During TD training and during the transfer tasks, there were three blocks of 10 stimulus presentations each per session. Within each block, $\mathrm{S}+$ and $\mathrm{S}-$ were each presented five times with no more than 3 consecutive minutes of any one stimulus. A VI 1-min schedule was in effect during $\mathrm{S}+$ periods only.

The ND group received the same sequence of the two training stimuli as the TD group, but the stimuli were both paired with the VI 1-min schedule of reinforcement. The SS group was presented with only the vertical white line paired with VI 1-min reinforcement. Training under all conditions was continued for 15 sessions. Then half of the subjects from each of the TD, ND, and SS groups were randomly assigned to one of two subgroups. Subgroup S-2 was tested on the target bright-dim discrimination in Stage 2, whereas Subgroup S-4 was tested on the bright-dim task in Stage 4 following exposure to a wavelength discrimination (Stage 2 for this subgroup) and a return to the original TD, ND, and SS training conditions (Stage 3).

Stage 2. During Stage 2, Subgroup S 4 was given discrimination training with $555 \mathrm{~nm}$ as $\mathrm{S}+$ and $538 \mathrm{~nm}$ as $\mathrm{S}-$, whereas for the Subgroup S-2 the bright cue was $S+$ and the dim was $S-$. During both discrimination tasks, the white vertical line was continuously displayed on the pecking key. Twenty daily sessions of discrimination training were provided in both Stages 2 and 4.

Stage 3. Following completion of Stage 2, Subgroup S-4 subjects, which had been tested with the wavelength discrimination problem in Stage 2, were returned to their respective Stage 1 training conditions for 10 sessions.

Stage 4. Subgroup S 4 subjects in the TD, ND, and SS conditions were given 20 sessions of discrimination training with the brightness task, under conditions identical to those experienced by the Subgroup S-2 subjects during Stage 2. During the first five sessions of the bright-dim task, data collection was arranged so that rate of responding was recorded for $\mathrm{S}+$ and $\mathrm{S}-$ over blocks of 10 trials including 5 of each type.

\section{RESULTS}

\section{Stage 1}

The TD procedure was effective in yielding good discriminative performance, with a mean of $82 \%$ of total responses emitted to $\mathrm{S}+$ on the last day of Stage 1 training. The ND group gave $53 \%$ of their total responses to the vertical line stimulus.

The average numbers of total responses emitted to the S+ on the last session for the TD, ND, and SS groups were $1,598.1,994.3$, and 926.6, respectively $[F(2,45)=14.81, p<.01]$. The total responses for each SS trained subject were divided by two, since they were exposed to the S+ for twice as long as the other subjects.

\section{Stage 2 (Subgroup S-4)}

The wavelength transfer problem proved to be exceedingly difficult for the subjects in this experiment. At the completion of 20 sessions of training, asymptotic performance reflected by the mean of the last 3 sessions averaged approximately $70 \%$ of total 
responses emitted to the $\mathrm{S}+$ value. Although the improvement measured over the first 15 blocks of training trials was statistically significant $[F(14,294)=26.87$, $\mathrm{p}<.01]$ there was no group difference between TD, $\mathrm{ND}$, and SS in acquisition or at asymptote, and no significant Group by Block interaction. Although the wavelength stimuli were the same ones used by Eck et al. (1969), in the present study a houselight was used, which would serve to desaturate the on-key stimuli and make them less discriminable.

\section{Stage 3 (Subgroup S-4)}

In Stage 3 the subjects in the TD, ND, and SS conditions performed similarly to the way they had in Stage 1. During the final session in Stage 3, the TD group gave $81 \%$ of its total responses to the vertical line St; the ND group averaged $51 \%$. The average number of responses emitted to the S+ during the last day of Stage 3 for the TD, ND, and SS trained pigeons was $1,533.9,1,147.4$, and 1,166.0, respectively. These values were compared to those for the TD, ND, and SS groups of Subgroup S-2, and in no cases were the differences significant $(F s<1)$. Thus, any possible differences in discriminative performance between the subjects which received the brightness task in Stage 2 and those which experienced it in Stage 4 could not be due
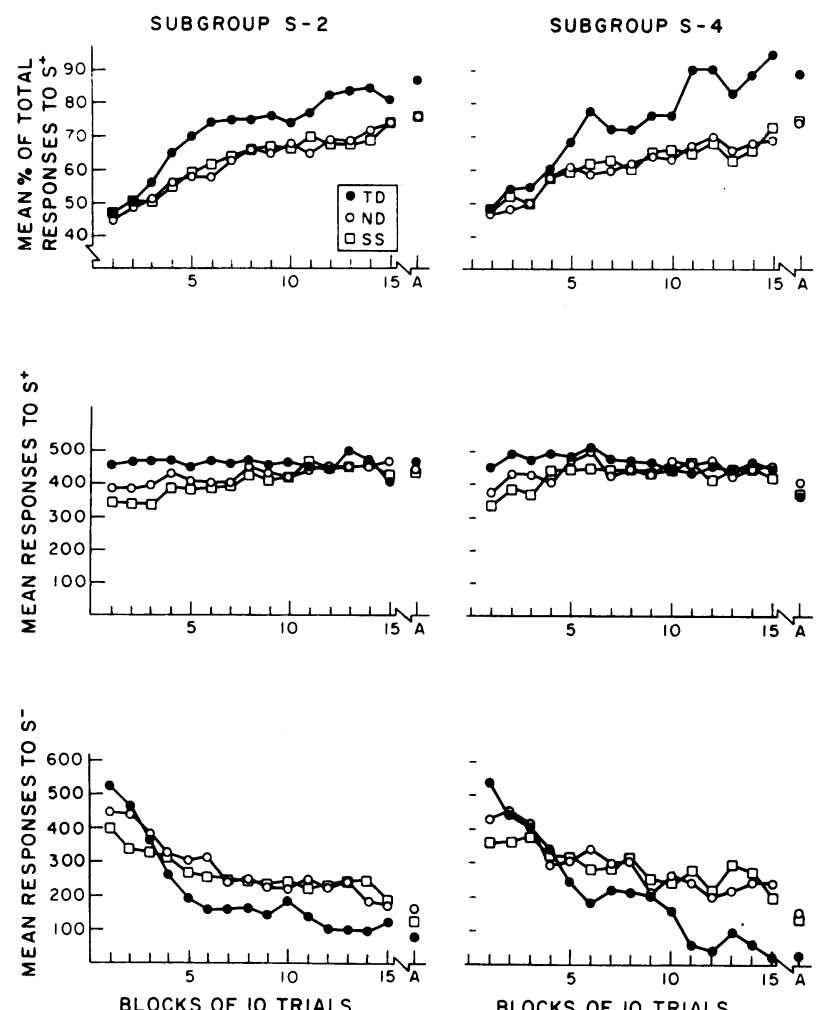

Figure 1. Discrimination performance of TD, ND, and SS groups as a function of prior exposure to another transfer problem. to different levels of performance in the immediately prior training stage.

\section{Stages 2 and 4 (The Brightness Transfer Task for Both Subgroups)}

In the upper left panel of Figure 1 is a plot of the mean percent of total responses to S+ for the Subgroup S-2 . Asymptotic discriminative performance, as reflected in the average over Sessions 18-20, is presented above the " $A$ " on the abscissa of each panel of Figure 1. In the upper right panel are the comparable data for the Subgroup S4. Whether the brightness task was the first or the second transfer problem experienced seems to make very little difference; there is a TD facilitation effect which is evident both in acquisition and at asymptote, but there is no suggestion of an ND retardation effect with either measure.

An analysis of variance of the acquisition data (Blocks 1-15) indicated that there was no reliable effect of sequence (Subgroup S-2 vs. S-4) or reliable interaction involving sequence. There was, however, a significant effect of blocks $[F(14,588)=49.32, p<.01]$, groups $[F(2,42)=4.46, p<.02]$, and the Groups by Blocks interaction $[\mathrm{F}(28,588)=2.16, \mathrm{p}<.01]$.

With regard to the measure of asymptotic performance, analysis of variance indicated no reliable sessions effect across the last three sessions, which is consistent with our description of these data as asymptotic. The analysis revealed only a significant effect of group (TD, ND, and SS) $[\mathrm{F}(2,42)=6.06, \mathrm{p}<.01]$. As is evident from the figure, the TD groups had a higher discrimination index than the ND and SS groups, which did not differ.

Both the absence of a sequence effect and the comparability of the ND and SS groups is further illustrated in the two middle panels of Figure 1, which report mean total responses to $\mathrm{S}+$, and the lower panels, which present mean total responses to $S-$. The TD groups had a higher rate of response to the vertical line in the preceding stage, and continue to respond more rapidly to St at the start of the brightness task. However, the response rates in the other groups increased and came to equal the rate of the TD group, after which no further changes in S+ responding took place. Analysis of variance indicated a significant blocks effect $[\mathrm{F}(14,588)=$ $4.74, \mathrm{p}<.01]$ and a significant Groups by Blocks interaction $[F(28,588)=2.25, \mathrm{p}<.01]$. The lower two panels in Figure 1 show a substantially more rapid extinction of responding to $S-$ in the TD groups. Again sequence, that is, the presence or absence of experience with a prior discrimination problem, has no consequence. Analysis of variance of the acquisition data for S- responding revealed a significant blocks effect $[F(14,588)=$ $40.95, \mathrm{p}<.01$ ] and a significant Groups by Blocks interaction $[F(28,588)=4.32, p<.01]$. Even at asymptote, there was a significant effect of groups $[F(2,42)=$ $4.06, \mathrm{p}<.02]$, with the TD groups responding at the lowest level. 


\section{DISCUSSION}

In Stage 2 of the Eck et al. (1969) experiment, in which a wavelength transfer problem was employed, evidence was obtained for a TD facilitation effect but not for an ND retardation effect. Furthermore, the TD facilitation effect was localized in more rapid extinction of responding to $S$-. In the present experiment, despite a large number of procedural differences including the use of an off-key brightness transfer task, we have replicated that pattern of results exactly. We have also extended the Eck et al. finding by showing that the TD facilitation effect extends beyond accelerated acquisition of a novel discrimination and includes achievement of a higher asymptotic level of performance. Furthermore, the failure of Eck et al. to find an ND retardation effect is not due to the lack of sufficient exposure to the transfer problem since, in the present study, the comparability of SS and ND groups continued until both groups had achieved their asymptotic performance levels. Neither was it due to an insufficient amount of training in Stage 1. Our procedure employed ND training rather than PD training. ${ }^{1}$ Thomas and Wheatley (1974) had shown that ND and PD procedures have comparable flattening effects on generalization gradients. We may now infer that this comparability extends to the transfer of training paradigm.

The failure to find an ND retardation effect remains an embarrasment to the general attention position. It may indicate that a different mechanism underlies the effects of TD and ND training on generalization slope and on nonspecific transfer. However, several considerations suggest that this conclusion may be premature. All of the transfer studies thus far reported have employed an extradimensional design in which the training stimuli used in Stage 1 were within a dimension and orthogonal to that involved in Stage 2. Even with the generalization slope measure, in the extradimensional paradigm the flattening effect of ND training is slight compared to the more robust TD sharpening effect. Tomie, Davitt, and Thomas (1973) have reported that the flattening of generalization by interdimensional ND training is greater than that following extradimensional ND training. Presumably, learning about stimulus insignificance is enhanced to the degree that nondifferentially reinforced stimuli are as dissimilar as possible. On the other hand, the same authors (Tomie, Davitt, \& Thomas, 1975) reported that the sharpening of generalization is greatest following extradimensional TD training. This suggests the intriguing possibility that, if a transfer study were done with highly dissimilar stimuli in Stage 1 , an ND retardation effect might be obtained along with a negligible TD facilitation effect.

One additional factor may also contribute to the difference between generalization slope and transfer results. Generalization testing in extinction is biased toward showing an ND effect in the sense that stimuli really do not matter, that is, they are all nonreinforced. Thus, the test experience may confirm the expectancy of stimulus insignificance created by the prior ND training. A transfer test, on the other hand, is biased toward showing a TD effect since, in testing, stimuli do matter, that is, they are differentially reinforced. Thus, the test experience may disconfirm the expectancy of stimulus insignificance which ND subjects bring to the transfer task. Subsequent attempts to demonstrate ND retardation effects in transfer designs might be well advised to select transfer tasks which are less biased toward invalidating expectancies resulting from the ND training experience. Possible examples would be multiple schedules involving reinforcement in both components but delivered according to different contingencies, that is, VI VT (variable time) or VI VR (variable ratio).

\section{REFERENCES}

Bresnatan, E. L. Effects of extradimensional pseudodiscrimination and discrimination training upon stimulus control. Journal of Experimental Psychology, $1970,85,155-156$.

Catania, A. C., \& Reynolds, G. S. A quantitative analysis of the responding maintained by interval schedules of reinforcement. Journal of the Experimental Analysis of Behavior, 1968, 11, 327-383.

EcK, K. O., Noel, R. C., \& Thomas, D. R. Discrimination learning as a function of prior discrimination and nondifferential training. Journal of Experimental Psychology, 1969, 82, 156-162.

Frieman, J., \& GoYette, C. H. Transfer of training across stimulus modalities and response class. Journal of Experimental Psychology, 1973, 97, 235-241.

Hickis, C. F., Robles, L., \& Thomas, D. R. Contextual stimuli and memory retrieval in pigeons. Animal Learning \& Behavior, in press.

Honig, W. K. Attentional factors governing the slope of the generalization gradient. In R. $M$. Gilbert \& N. $S$. Sutherland (Eds.), Animal discrimination learning. London: Academic Press, 1969.

Thomas, D. R., Freeman, F., Svinicki, J. G., BurR, D. E. S., \& Lyons, J. The effects of extradimensional training on stimulus generalization. Journal of Experimental Psychology, 1970, 83(1, Part 2).

Thomas, D. R., \& Wheatley, K. L. Effects of interdimensional training on stimulus generalization: An extension. Journal of Experimental Psychology, 1974, 103, 1080-1085.

Tomie, A., Davitt, G. A., \& Thomas, D. R. Role of stimulus similarity in equivalence training. Journal of Experimental Psychology, 1973, 101, 146-150.

Tomie, A., Davitt, G. A., \& Thomas, D. R. Effects of stimulus similarity in discrimination training upon wavelength generalization in pigeons. Journal of Comparative and Physiological Psychology, 1975, 88, 945-954.

\section{NOTE}

1. Thomas et al. (1970) employed a special class of ND procedures called pseudodiscrimination (PD), in which the same distribution of reinforcement present in TD training was maintained in this particular ND procedure. Both ND and PD training have been shown to produce equivalent effects on generalization gradients relative to SS controls (Thomas \& Wheatley, 1974).

(Received for publication April 21, 1977.) 